\title{
Bacterial membrane activity of $\alpha$-peptide/ $\beta$-peptoid chimeras: Influence of amino acid composition and chain length on the activity against different bacterial strains
}

Line Hein-Kristensen ${ }^{1 *}$, Kolja M Knapp ${ }^{2}$, Henrik Franzyk ${ }^{2}$ and Lone Gram

\begin{abstract}
Background: Characterization and use of antimicrobial peptides (AMPs) requires that their mode of action is determined. The interaction of membrane-active peptides with their target is often established using model membranes, however, the actual permeabilization of live bacterial cells and subsequent killing is usually not tested. In this report, six $\alpha$-peptide/ $\beta$-peptoid chimeras were examined for the effect of amino acid/peptoid substitutions and chain length on the membrane perturbation and subsequent killing of food-borne and clinical bacterial isolates.

Results: All six AMP analogues inhibited growth of twelve food-borne and clinical bacterial strains including Extended Spectrum Beta-Lactamase-producing Escherichia coli. In general, the Minimum Inhibitory Concentrations (MIC) against Gram-positive and -negative bacteria were similar, ranging from 1 to $5 \mu \mathrm{M}$. The type of cationic amino acid only had a minor effect on MIC values, whereas chain length had a profound influence on activity. All chimeras were less active against Serratia marcescens (MICs above $46 \mu \mathrm{M}$ ). The chimeras were bactericidal and induced leakage of ATP from Staphylococcus aureus and S. marcescens with similar time of onset and reduction in the number of viable cells. EDTA pre-treatment of S. marcescens and E. coli followed by treatment with chimeras resulted in pronounced killing indicating that disintegration of the Gram-negative outer membrane eliminated innate differences in susceptibility. Chimera chain length did not influence the degree of ATP leakage, but the amount of intracellular ATP remaining in the cell after treatment was influenced by chimera length with the longest analogue causing complete depletion of intracellular ATP. Hence some chimeras caused a complete disruption of the membrane, and this was parallel by the largest reduction in number of viable bacteria.

Conclusion: We found that chain length but not type of cationic amino acid influenced the antibacterial activity of a series of synthetic $\alpha$-peptide/ $\beta$-peptoid chimeras. The synthetic chimeras exert their killing effect by permeabilization of the bacterial cell envelope, and the outer membrane may act as a barrier in Gram-negative bacteria. The tolerance of $S$. marcescens to chimeras may be due to differences in the composition of the lipopolysaccharide layer also responsible for its resistance to polymyxin B.
\end{abstract}

\footnotetext{
*Correspondence: lihe@food.dtu.dk

'Division of Industrial Food Research, National Food Institute, Technical

University of Denmark, Søltofts Plads, bldg.221, 2800 Kgs. Lyngby,

DK-Denmark

Full list of author information is available at the end of the article
} 


\section{Background}

Antimicrobial peptides (AMPs) are host defence molecules that constitute an essential part of the innate immune system among all classes of life [1]. Most AMPs permit the host to resist bacterial infections by direct killing of invading bacteria or other microorganisms, however, many AMPs are also immuno-modulatory and thus enhance the host defence against pathogens [2-5].

In addition to their natural role in combating infections, AMPs are recognized as promising alternatives to conventional antibiotics for which development of resistance has become an ever-increasing concern [6-8]. Peptide based drugs are often hampered by a rapid in vivo degradation, however, this may be circumvented by stabilizing natural AMPs by single-site substitutions or by designing novel synthetic analogues with an altered backbone that confers complete stability to the compounds. Careful investigation of structure-activity relationships may eventually allow design of optimised antimicrobial compounds with high activity and minimal side effects [9-15].

Many AMPs fold into an amphipathic structure, and it is believed that this topology enables pore formation or disintegration of bacterial cell membranes leading to bacterial cell death. The amphipathic properties usually include cationic patches that promote interaction with the anionic bacterial membrane as well as hydrophobic patches that favor integration into the membrane. Since this is the most common mode of action for AMPs there has been an intense focus on their ability to adapt an amphipathic conformation [16,17]. In particular, design of peptides with a high propensity to fold into a helical amphipathic conformation has attracted considerable interest [13,18-20].

We have previously described a synthetic approach for design of $\alpha$-peptide/ $\beta$-peptoid chimeras possessing a design with alternating $\mathrm{N}$-alkylated $\beta$-alanine ( $\beta$-peptoid) and $\alpha$-amino acid units (Figure 1 ). In addition, preliminary investigations showed that such peptidomimetics constitute a novel subclass of proteolytically stable antimicrobial compounds [21-23]. This design displays chiral unnatural $\beta$-peptoid residues that appear to contribute with structure-promoting effects and lipophilicity, while strongly cationic properties and intramolecular hydrogen bonding capacity are introduced via the $\alpha$-amino acids lysine and/or homoarginine [24]. The precise secondary structure of these chimeras still remains to be elucidated, nevertheless, circular dichroism (CD) spectroscopy clearly indicates the presence of some degree of secondary structure $[22,23]$. Interestingly, a higher degree of secondary structure was found for analogues containing chiral side chains in the $\beta$-peptoid units (i.e. compounds 2 and 3 in Figure 1) as compared to chimeras with achiral $\beta$-peptoid residues (i.e. compound 1 in Figure 1) [22], but<smiles>[R]C(c1ccccc1)N(CCC(N)=O)C(=O)[C@H](CCCCNC(=N)N)NC(C)=O</smiles>

$1 \mathrm{R}=\mathrm{H}$

$2 \mathrm{R}=\mathrm{Me}$<smiles>CC(=O)N[C@@H](CCCCN)C(=O)N(CCC(N)=O)[C@@H](C)c1ccccc1</smiles>

3<smiles>CCCCC[C@H](NC(C)=O)C(=O)N(CCC(=O)N[C@@H](CCCCNC(=N)N)C(=O)N(CCC(N)=O)[C@@H](C)c1ccccc1)[C@@H](C)c1ccccc1</smiles>

$$
\begin{aligned}
& \text { 4a } n=2 \\
& \text { 4b } n=3 \\
& \text { 4c } n=4
\end{aligned}
$$

Figure 1 Chemical structure of the six $\alpha$-peptide/ $\beta$-peptoid chimeras

the effect of this on antibacterial activity remains largely unresolved [23].

The membrane-destabilizing effects of the chimeras have only been investigated in model liposomes prepared from phosphatidylcholine, a phospholipid found 
predominantly in eukaryotic cells, and several of the chimeras permeabilized such liposomal membranes [24]. Most studies on membrane activity of antimicrobial peptides have in fact been performed on model membranes [25-28] while the effects on cell membranes of viable bacteria have often not been tested. Also, the effect of membrane permeabilization on killing of bacteria has not been tested [27].

Here, we test the antibacterial effect of six chimeras against a spectrum of bacterial strains that include several important clinical and food-borne pathogens. The main purpose was to examine how the type of cationic amino acid and sequence length affected the antibacterial activity and to correlate this to a potential membrane-related mode of action in viable bacteria.

Part of this work was presented at the $50^{\text {th }}$ InterScience Conference on Antimicrobial Agents and Chemotherapy in Boston $12-15^{\text {th }}$ of September 2010.

\section{Methods}

\section{Bacterial strains and culture conditions}

Initial activity experiments were carried out with twelve strains from seven bacterial species representing common laboratory strains and clinical strains derived from both food-borne and nosocomial infections (Table 1). Stock cultures were stored at $-80^{\circ} \mathrm{C}$ in $4 \%(\mathrm{w} / \mathrm{v})$ glycerol, $0.5 \%(\mathrm{w} / \mathrm{v})$ glucose, $2 \%(\mathrm{w} / \mathrm{v})$ skimmed milk powder and $3 \%(\mathrm{w} / \mathrm{v})$ tryptone soy powder. All experiments were carried out with bacteria incubated for one night (i.e. approximately 18 hours) at $37^{\circ} \mathrm{C}$. Experiments were performed in cation-adjusted Mueller Hinton II broth (MHB) (Becton Dickinson 212322) adjusted to $\mathrm{pH} 7.4$ or Tryptone Soy Broth (TSB) (Oxoid CM0129) for the

Table 1 Origin and reference of bacterial strains used in the present study

\begin{tabular}{|c|c|c|}
\hline & Origin & Ref \\
\hline S. aureus $8325-4$ & Wildtype & [59] \\
\hline K. pneumoniae ATCC 13883 & Human, clinical & - \\
\hline S. marcescens ATCC 8100 & Human, clinical & - \\
\hline E. coli ATCC 25922 & Wildtype & - \\
\hline E. coli MG1655 & K-12 F' lambda & [60] \\
\hline E. coli AAS-EC-009 & Human, clinical & a \\
\hline E.coli AAS-EC-010 & Human, clinical & a \\
\hline L. monocytogenes 4446 & Human, clinical & {$[61]$} \\
\hline L. monocytogenes N53-1 & Food processing & {$[62]$} \\
\hline L. monocytogenes EGD & Wildtype & $b$ \\
\hline V. vulnificus ATCC $^{\top}$ & Human, clinical & - \\
\hline V. parahaemolyticus ATCC ${ }^{\top}$ & Human, clinical & - \\
\hline
\end{tabular}

Susceptibility testing were carried out with a selection of twelve different bacterial strains comprising common laboratory strains and clinical strains derived from food-borne pathogens as well as pathogens responsible for nosocomial infections. ${ }^{a}$ ESBL-producing clinical samples from Danish patients in $2007 ;^{b}$ This strain was kindly provided by Werner Goebel, University of Würzburg.
ATP leakage assays. Brain Heart Infusion (BHI) (CM1135) with agar (VWR 20768.292) 1.5\% as gelling agent was used throughout for colony plating.

\section{Peptide synthesis and selection}

$\alpha$-Peptide/ $\beta$-peptoid chimeras consisting of alternating repeats of natural cationic $\alpha$-amino acids and synthetic lipophilic $\beta$-peptoid residues were prepared by solid-phase synthesis as previously described $[21,22]$. Six chimeras were investigated in this study. The possible differences in sensitivity of different bacterial species were evaluated by testing the analogues 1, 2 and 3, distinguished by different degrees of chirality and type of cationic amino acid. Additionally, the mixed series $4 \mathrm{a}, 4 \mathrm{~b}$ and $4 \mathrm{c}$, differing only in the chain length, was used for evaluating the effect of this on antimicrobial activity (Figure 1). Compounds 1, 2 and 3 have been described previously [23,24,29], while the series $4 \mathrm{a}, 4 \mathrm{~b}$ and $4 \mathrm{c}$ were synthesized using the already established synthesis protocols involving known dimeric building blocks [21,22]. The identity of the primary peptidomimetic sequences $4 \mathrm{a}, 4 \mathrm{~b}$ and $4 \mathrm{c}$ were confirmed by high-resolution MS (Bruker MicroTOF-Q LC mass spectrometer equipped with an electrospray ionization source): compound $4 \mathrm{a},(\mathrm{m} / \mathrm{z})[\mathrm{M}+4 \mathrm{H}]^{4+}$ obsd. $=339.9727(\mathrm{calcd} .=$ 339.9719, $\Delta \mathrm{M} 2.3 \mathrm{ppm})$; compound $4 \mathrm{~b},(\mathrm{~m} / \mathrm{z})[\mathrm{M}+5 \mathrm{H}]^{5+}$ obsd. $=402.0614($ calcd. $=402.0608, \Delta M 1.4$ ppm $) ;$ compound $4 \mathrm{c},(\mathrm{m} / \mathrm{z})[\mathrm{M}+6 \mathrm{H}]^{6+}$ obsd. $=443.2880($ calcd. $=$ 443.2879, $\Delta \mathrm{M} 0.2 \mathrm{ppm}$ ). Peptides were solubilized to a stock of $10 \mathrm{mg} / \mathrm{mL}$ in sterile MilliQ water and stored at $-20^{\circ} \mathrm{C}$.

\section{Determination of Minimum Inhibitory Concentration (MIC) and Minimum Bactericidal Concentration (MBC)}

The Minimum Inhibitory Concentration (MIC) of the chimeras was determined against the spectrum of bacteria using the microdilution method according to guidelines of the Clinical and Laboratory Standards Institute (CLSI) [30]. Chimera 1:2 serial dilutions were prepared from $1,024 \mu \mathrm{g} / \mathrm{mL}$ stock solutions to give a final range of $512-0.5 \mu \mathrm{g} / \mathrm{mL}$ in the wells. This corresponds to a final range of 144 to $0.14 \mu \mathrm{M}$ for the heaviest chimera (i.e. chimera 4c) and of 282 to $0.27 \mu \mathrm{M}$ for the lightest chimera (i.e. chimera 4a). Colonies grown overnight (i.e. approximately 18 hours) on BHI agar were suspended in $0.9 \%$ saline to give a turbidity of 0.13 at $\mathrm{OD}_{546}$ (approximately $1 \times 10^{8} \mathrm{CFU} / \mathrm{mL}$ ), and then diluted in $\mathrm{MHB}$ pH 7.4 to a final concentration of $5 \times$ $10^{5} \mathrm{CFU} / \mathrm{mL}$ in each well. Following CLSI guidelines the media for testing of Listeria monocytogenes strains were supplemented with $2.5 \%$ lysed horse blood. Polypropylene plates (Nunc 442587) were used to minimize peptide binding and incubation time was 18-20 hours at $37^{\circ} \mathrm{C}$. MIC was determined in a minimum of two technical replicates as the lowest concentration of the 
peptide analogue where no visible growth was found. The Minimum Bactericidal Concentration (MBC) was determined by plating $10 \mu \mathrm{L}$ of the suspension from the first three wells without growth on $\mathrm{BHI}$ agar and incubating these for 24 hours at $37^{\circ} \mathrm{C}$. $\mathrm{MBC}$ was the lowest concentration at which a $99.9 \%$ reduction in $\mathrm{CFU} / \mathrm{mL}$ was observed. Activity is expressed in $\mu \mathrm{mol} / \mathrm{L}$ to enable a direct comparison of analogues with different length $(=$ size $)$.

\section{Killing kinetics of Staphylococcus aureus and Serratia marcescens}

In vitro time-kill curves for chimera 1, 2 and 3 were determined against $S$. aureus 8325 (MIC $\mu \mathrm{M}$ : chimera 1 5.9; chimera 2 2.8; chimera 3 18.7) and Serratia marcescens ATCC 8100 (MIC $\mu \mathrm{M}$ : chimera 1 46.8; chimera 2 45.5; chimera 3 150.0). These two bacterial strains represent organisms susceptible and tolerant to the chimeras, respectively. The bactericidal effect of the three chimeras was tested at MIC in two independent experiments; additionally the effect of chimera 2 was tested at $1 / 4$ and $1 / 2$ times MIC. In brief, a suspension prepared from fresh overnight colonies as described above was transferred to $2 \mathrm{~mL}$ PBS or cation-adjusted MHB with chimera added (from a $10 \times$ MIC solution) to give a similar bacterial cell density as employed in the MIC determination; the resulting suspension was then incubated at $37^{\circ} \mathrm{C}, 300 \mathrm{rpm}$. Samples for colony determination were taken at $0,1,2,4,6$ and 8 hours after addition and transferred to a ten-fold dilution row. Colony counts were determined after incubation for 24 hours at $37^{\circ} \mathrm{C}$.

\section{ATP leakage assay}

Pore formation as caused by peptide addition was determined by measuring ATP leakage from the bacterial cell using a bioluminescence assay [31]. The assay was used to estimate differences between sub-typical chimeras 1, 2 and 3 on $S$. aureus and $S$. marcescens and to evaluate the effect of chain length of mixed type chimeras $4 \mathrm{a}, 4 \mathrm{~b}$ and $4 c$ on $S$. aureus. In brief, bacteria were grown in TSB at $37^{\circ} \mathrm{C}$ for 24 hours and then re-inoculated in TSB at $37^{\circ} \mathrm{C}$ for 6-8 hours until an absorbance at $546 \mathrm{~nm}$ of 2.5 for $S$. aureus and 2.0 for $S$. marcescens and then harvested $(10 \mathrm{~min}$ at $2,000 \times \mathrm{g})$. The bacteria were grown to a high absorbance since a high concentration of bacteria was necessary in order to get a measurable response in the ATP leakage assay. Cells were washed once in $50 \mathrm{mM}$ potassium phosphate buffer $(\mathrm{pH} 7.0)$ and once in $50 \mathrm{mM}$ HEPES buffer ( $\mathrm{pH} 7.0$ ), before the pellet was resuspended in HEPES buffer to an $\mathrm{OD}_{546} \sim 10$, and then stored on ice. Before chimera addition bacteria were pre-incubated with $0.2 \%(\mathrm{w} / \mathrm{v})$ glucose to energize the cells. In general a chimera dose of $1000 \mu \mathrm{g} / \mathrm{mL}$ (corresponding to
280-552 $\mu \mathrm{M}$ for all chimeras) was used for all assays; however, for determining dose response curves additional doses of $100(28-55 \mu \mathrm{M}), 250(71-137 \mu \mathrm{M})$ and 500 $(140-276 \mu \mathrm{M}) \mu \mathrm{g} / \mathrm{mL}$ were tested, and only the immediate release was noted. Total ATP and extracellular ATP were determined with a luminometer (Pharmacia Biotech Novaspec II Visible Spectrophotometer). Intracellular volumes [32] of S. aureus and S. marcescens $\left(0.85 \mu \mathrm{m}^{3}\right.$ and $1.7 \mu \mathrm{m}^{3}$, respectively) were subtracted from the total volume before calculating the extracellular ATP concentration; the intracellular ATP concentration could then be calculated from this and the total ATP. ATP leakage kinetics was determined on a bacterial suspension prepared as above. Samples were taken at time 0, 5, 10, 20, 30 and 60 minutes and viable counts determined. Both the ATP leakage assay and killing kinetics performed under the same assay conditions were performed in two independent experiments.

\section{Results}

Based on our previously published work on $\alpha$-peptide/ $\beta$-peptoid chimeras $[23,24,29]$ we selected six compounds for the present study. Our main purpose was to examine the influence of the type of cationic amino acid and chain length on antibacterial activity and specificity. Also we aimed at elucidating the mechanism of action against live bacterial cells and determine if this (membrane perturbation) was influenced by the chimera structural characteristics. We measured ATP leakage from chimera-treated cells as an indication of membrane pertubation. Comparing the ATP leakage with time-kill studies allowed us to establish if there was a direct correlation between permeabilization of the membrane and killing of bacterial cells.

\section{MIC and MBC against clinical and food-borne pathogens}

Twelve strains representing seven bacterial species were tested for their susceptibility to the peptide analogues. The analogues exhibited a broad-spectrum activity with no distinct differences between Gram-positive and -negative bacteria (Table 2). Five of the six chimeras had a strong antibacterial effect with MIC values below $5 \mu \mathrm{M}$. Important food-borne pathogens were included in the susceptibility assay panel. Thus, three L. monocytogenes strains representing both a clinical lineage 1 strain (strain 4446) and a persistent lineage 2 strain from a food-processing plant (strain N53-1) as well as clinical isolates of $V$. vulnificus and $V$. parahaemolyticus were examined.

The MIC values of chimeras 1, 2 and 3 were similar, indicating that the $\beta$-peptoid side chain chirality (i.e. 1 vs. 2) had no effect on antibacterial activity and that the 12meric homoarginine (hArg) based sequence 2 was likely equalled by the longer 16-meric lysine-containing analogue 3. Generally, low MIC values were found for these 
Table 2 Minimum Inhibitory Concentration $(\mu \mathrm{M})$ of the six $\alpha$-peptide/ $\beta$-peptoid chimeras in the present study

\begin{tabular}{|c|c|c|c|c|c|c|}
\hline & Chimera 1 & Chimera 2 & Chimera 3 & Chimera 4a & Chimera 4b & Chimera $4 c$ \\
\hline S. aureus 8325 & 5.9 & 2.8 & 18.7 & 141.2 & 23.8 & 4.5 \\
\hline K. pneumoniae ATCC 13883 & 1.5 & 2.8 & 37.5 & 282.4 & 23.8 & 9.0 \\
\hline S. marcescens ATCC 8100 & 46.8 & 45.5 & 150.0 & $>282.4$ & 190.3 & 71.8 \\
\hline E. coli ATCC 25922 & 1.5 & 2.8 & 9.4 & 141.2 & 3.0 & 2.2 \\
\hline E. coli MG1655 & 1.5 & 2.8 & 4.7 & 141.2 & 5.9 & 2.2 \\
\hline E. coli AAS-EC-009 & 1.5 & 2.8 & 9.4 & 141.2 & 11.9 & 4.5 \\
\hline E.coli AAS-EC-010 & 1.5 & 1.4 & 9.4 & 141.2 & 3.0 & 2.2 \\
\hline L. monocytogenes 4446 & 2.9 & 1.4 & 1.1 & 70.6 & 3.0 & 1.1 \\
\hline L. monocytogenes N53-1 & 2.9 & 2.8 & 1.1 & 70.6 & 5.9 & 1.1 \\
\hline L. monocytogenes EGD & 1.5 & 2.8 & 1.1 & 70.6 & 3.0 & 1.1 \\
\hline V. vulnificus $\mathrm{ATCC}^{\top}$ & 1.5 & 1.4 & 2.3 & 35.3 & 3.0 & 2.2 \\
\hline V. parahaemolyticus ATCC ${ }^{\top}$ & 1.5 & 1.4 & 2.3 & 70.6 & 3.0 & 1.1 \\
\hline
\end{tabular}

Minimum Inhibitory Concentration of the six peptidomimetics in this study against the spectrum of bacteria expressed in $\mu \mathrm{M}$. Values were obtained from a minimum of two independent trials. The Minimum Bactericidal Concentration (MBC) was in all assays equal to or a maximum of one two-fold higher than the MIC value indicating a bactericidal mode of action.

three compounds, however, the activity of chimera 3 was slightly lower than for chimera 1 and 2 against some of the bacteria i.e. S. aureus, K. pneumoniae and $S$. marcescens.

Chimeras 4a, 4b and 4c all have a 1:1 mixture of Lys and hArg residues, but differ in length (8-16 residues), and this had a marked effect on their antibacterial activity. The pattern was the same against all bacterial strains tested. The longest of the three, chimera 4c, was the most active compound with MIC values of 1.1-2.2 $\mu \mathrm{M}$ against the foodborne pathogens L. monocytogenes and Vibro spp. Chimera $4 \mathrm{c}$ was also active against the clinical strains of $E$. coli, $S$. aureus and K. pneumoniae with MIC values in the range of 2.2-9.0 $\mu \mathrm{M}$ (Table 2). Chimera $4 \mathrm{~b}$, with a length of 12 residues, was less antibacterial with MIC values approximately 2-3 times higher than those of the 16-mer 4c (Table 2). Chimera 4a being only half the length of chimera $4 \mathrm{c}$ was the least antibacterial as the MIC values were 15-70 times higher than those of chimera 4c (Table 2). Thus, the relative increase in activity was much larger for elongation with a third repeating unit (i.e. from 8-mer 4a to 12 -mer $4 \mathrm{~b}$ ), than the further elongation of $4 \mathrm{~b}$ with a fourth repeating unit to afford 4c, revealing the minimally required length of an active AMP analogue to be approximately 12 residues.

Two Extended Spectrum Beta-Lactamase (ESBL)-producing E. coli clinical isolates (AAS-EC-009 and AASEC-010) were included to determine if this antibiotic resistance affected chimera sensitivity. However, the chimeras were as effective against these strains as against non-ESBL strains indicating that resistance mechanisms conferring resistance to conventional antibiotics do not diminish the activity of the present peptidomimetics. Interestingly, S. marcescens, which is known to be intrinsically resistant to other antimicrobial peptides, was tolerant to all six chimeras (MICs above $46 \mu \mathrm{M}$; Table 2), and it most likely possesses resistance mechanisms that are different from those present in the two multi-resistant $E$. coli strains.

All six chimeras had a Minimum Bactericidal Concentration $(\mathrm{MBC})$ equal to or double the MIC. The high similarity between the MIC and MBC values indicates that the chimeras exhibit a bactericidal mode of action.

Killing kinetics in two bacteria with different susceptibility $S$. marcescens was the only bacterial strain tested that was tolerant to the $\alpha$-peptide $/ \beta$-peptoid chimeras. The strain is the only one considered intrinsically resistant to the polymyxin group of AMPs, and this could explain its resistance to our peptidomimetics. If so, this would indicate that a very similar resistance mechanism was responsible for the observed decrease in susceptibility. Therefore we performed a comparative mechanistic study that also included $S$. aureus and E. coli as susceptible reference strains.

We exposed $S$. aureus and S. marcescens to peptidomimetics 1, 2 and 3 at three different concentrations in $\mathrm{MHB}$ as well as at their MIC concentration in PBS buffer in order to determine whether these chimeras were only active against growing bacterial cells. S. marcescens was killed rapidly by chimera 2 (Figure $2 \mathrm{~A}$ ), and the lethal effect was clearly concentration-dependent (Figure 2C). In contrast, $S$. aureus was killed more slowly and with a less pronounced effect of dose (Figure 2B and 2D). Treatment of $S$. marcescens with chimera 2 at its MIC caused a $2 \log$ decrease in the number of viable bacteria within the first hour after which cell numbers declined over the next 5 hours. When the bacteria were treated with the chimera in PBS, the killing occurred very rapidly and no viable cells remained after the first hour. When $S$. aureus was treated with chimera 2 at the MIC in MHB, the number of viable cells did not decrease until after 6 
A

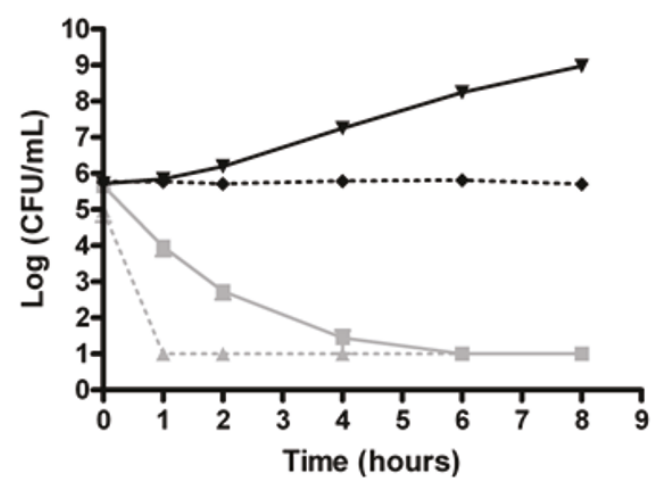

C

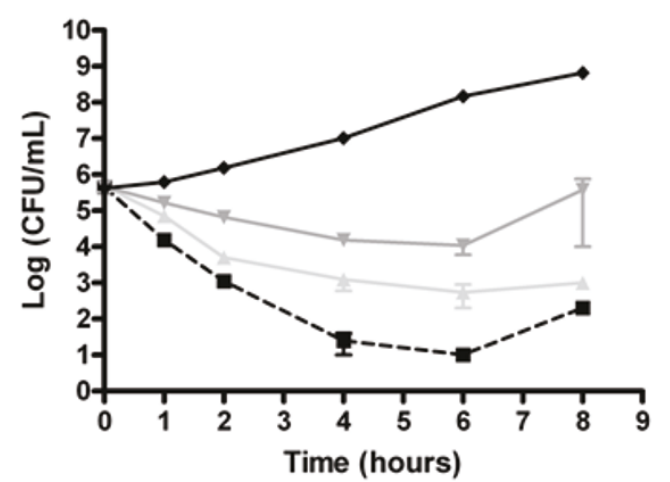

B

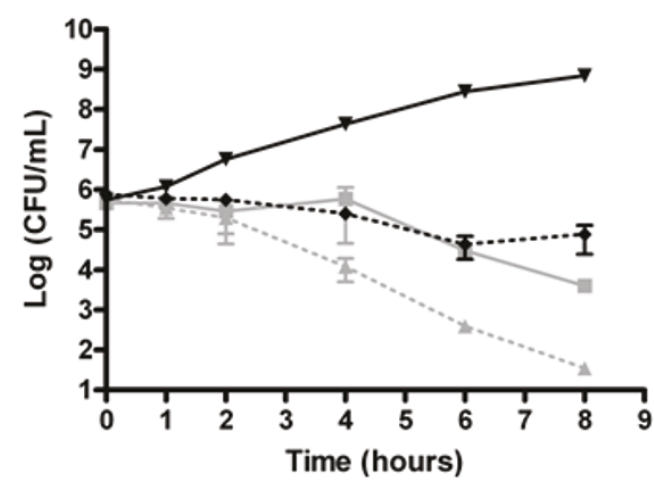

D

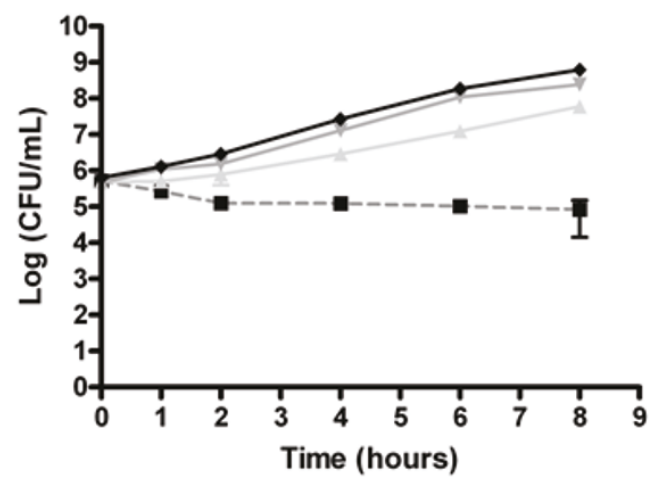

Figure 2 Killing kinetics of chimera 2 against S. marcescens $(A+C)$ and S. aureus $(B+D)$ displayed as mean number of viable cells with standard error of the mean (SEM). The assays were performed in two independent experiments. Time-kill of the chimera was determined at MIC in MHB (grey solid) and PBS (grey punctuated) and compared to MilliQ-treated control in MHB (black solid) and PBS (black punctuated) for S. marcescens (A) and S. aureus (B). The effect of chimera concentration on time-kill was determined in MHB at $1 / 4$ MIC (dark grey), $1 / 2$ MIC (light grey) and MIC (black punctuated) and compared with MilliQ-treated control (black solid) for S. marcescens and (C) and S. aureus (D).

hours, however, when treated in PBS, viable cell numbers decreased with $\log 2$ after 4 hours (Figure 2B). Even though a slightly decreased growth rate was observed for $S$. aureus upon treatment with concentrations below MIC as compared to the control, a concentration close to the MIC value was needed to completely inhibit growth of the culture (Figure 2D). In comparison, as low as $1 / 4$ MIC resulted in a reduction in cell number of $S$. marcescens (Figure 2C) revealing a more pronounced concentration-dependent killing for this bacterium.

Since the MIC value found for $S$. marcescens was considerably higher than that seen for $S$. aureus, we performed time-kill on E. coli, which exhibited a similar susceptibility in terms of MIC to that of S. aureus, to test if the rapid lethal effect against $S$. marcescens was due to the higher concentrations of peptidomimetics (E. coli ATCC 25922 MIC $\mu \mathrm{M}$ : chimera 1 1.5; chimera 2 2.8; chimera 3 9.4). However, a rapid killing effect was also found for this bacterial species (data not shown) ruling out that the elevated concentrations solely could be responsible for the high killing rate seen for $S$. marcescens.

\section{Membrane perturbation effects in two bacteria with different sensitivity}

Killing kinetics often reflect the mode of action, and we hypothesized that differences between $S$. aureus and $S$. marcescens regarding their sensitivity and time-kill might be due to different modes of interaction with the peptidomimetics. Therefore, an ATP bioluminescence assay was employed to determine (i) whether cell envelope perturbation was involved in the antibacterial effect, and (ii) if so, whether the organisms differed in the degree of ATP leakage.

Chimera 1, 2 and 3 caused leakage of ATP from both $S$. aureus and $S$. marcescens, but all three peptidomimetics gave rise to an ATP leakage from S. aureus that was substantially larger than that from S. marcescens (see Figure 3 for results with chimera 1). The intracellular ATP concentration rapidly approached zero for both bacteria within the first few minutes, whereas the extracellular ATP concentration increased more rapidly during the first minutes for $S$. aureus $(\sim 20 \mu \mathrm{M})$ than for $S$. marcescens $(\sim 5 \mu \mathrm{M})$. To examine if this could be due to the fact 
that the two bacteria were treated with the same dose despite their very different MIC values, we determined their dose response curves. For both bacteria a minimum chimera dose of $500 \mu \mathrm{g} / \mathrm{mL}$ (i.e. 145-180 $\mu \mathrm{M}$ ) was needed to obtain the maximum immediate response (data not shown) ruling out that the rapid release of ATP from $S$. aureus seen in Figure 3A is due to a higher concentration/MIC ratio than employed for $S$. marcescens.

To investigate if the degree of ATP leakage from the bacterial cell corresponded to the simultaneous decrease in the number of viable cells (i.e. if $S$. marcescens cells on the basis of their elevated MIC were in fact able to survive even after a moderate ATP leakage) we determined time-kill under exactly the same conditions as the ATP bioluminescence assay had been performed. Irrespective of which of the three chimeras that were used, both bacteria were reduced 2-3 log from an initial value of $\log$ $\sim 9.5$ per $\mathrm{mL}$ within the first 20 minutes before the ATP leakage tailored off and no further decrease in viable count was seen for up to 60 minutes (not shown). This indicates that the degree of ATP leakage from the two bacteria (i.e. the concentration of the extracellular ATP) does not reflect differences in viability. No reduction in the number of viable bacteria was seen for the control (not shown), and the intracellular concentration of ATP did not change (Figure $3 \mathrm{~A}$ and $3 \mathrm{~B}$ ).

Although there was no systematic difference in the MIC values between Gram-positive and -negative bacteria, we speculated that the Gram-negative outer membrane could act as a barrier to the penetration of AMPs, since polymyxin B resistance in $S$. marcescens has been linked to induced changes in the amount and composition of lipopolysaccharide (LPS) in the outer membrane [33]. Moreover, similar resistance-conferring membrane alterations have also been seen for other bacteria in response to polymyxin B treatment [34-36]. Accordingly, we studied how a membrane-destabilizing pre-treatment of $S$. marcescens, E. coli and S. aureus with the divalent metal cation-chelating agent EDTA would affect the killing caused by chimera 1 . In these experiments we used a non-lethal $0.5 \mathrm{mM}$ concentration of EDTA together with the non-lethal $1.5 \mu \mathrm{M}$ concentration of the tested AMP analogue. A slight reduction in the number of viable cells corresponding to $0.5 \log$ was seen for $S$. aureus when treated with chimera 1 alone while $E$. coli and $S$. marcescens were reduced with $1.5 \log$ (data not shown). No discernable difference in the number of viable cells remaining was observed between $S$. aureus treated successively with EDTA and peptidomimetic and S. aureus treated only with the peptidomimetic. In contrast, cell numbers of both $S$. marcescens and $E$. coli were reduced with 4-5 log from an initial value of $\log \sim 5.5$ within the first 4 hours (not shown) upon treatment with a sublethal EDTA concentration together with the chimera. This indicates that the intact outer membrane indeed appears to act as a protective barrier against the antibacterial chimeras.

\section{The effect of chimera chain length on membrane perturbation activity}

Peptidomimetics $4 \mathrm{a}, 4 \mathrm{~b}$ and $4 \mathrm{c}$ consist of the same repeating unit of four residues (Figure $1 ; \mathrm{n}=2,3$ and 4, respectively), and thus differ only in length. The MIC values increased dramatically when going from 8-mer (4a) to 12 mer (4b) while further elongation to 16-mer (4c) only led to a slight enhancement in potency (Table 2). Hence, we were intrigued to establish whether mechanistic differences could explain this strong correlation.
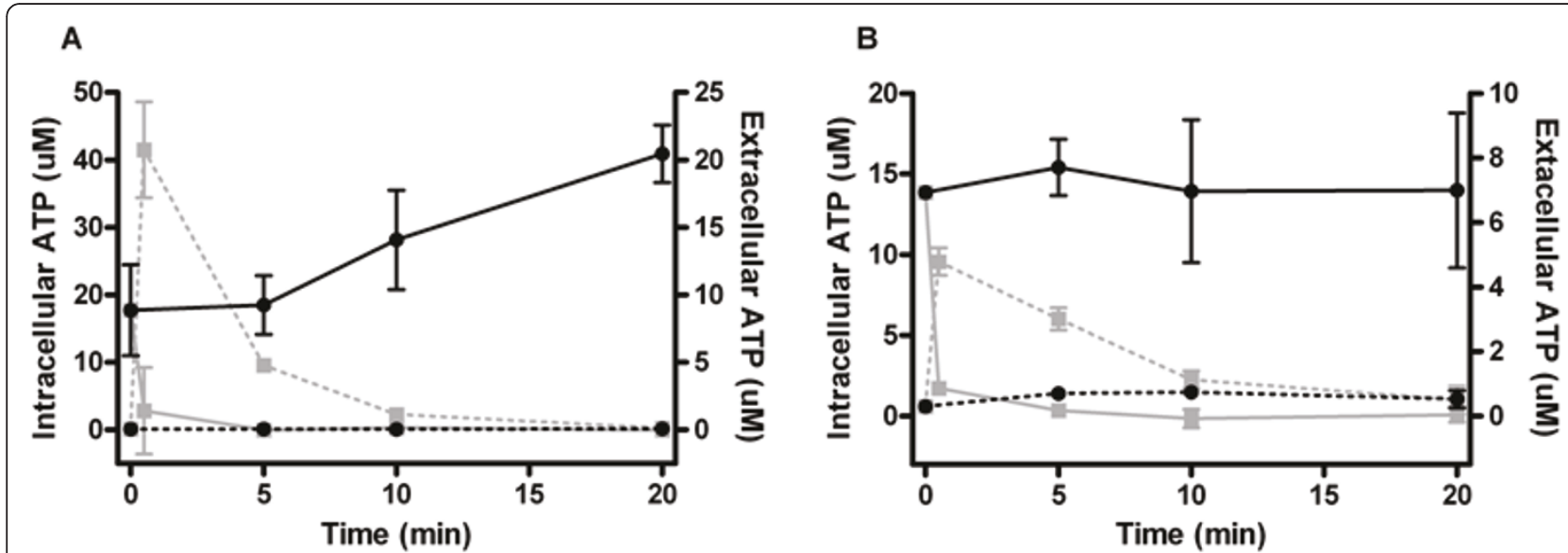

Figure 3 Chimera-induced ATP leakage in S. aureus (A) and S. marcescens (B) after treatment with $1000 \mu \mathrm{g} / \mathrm{mL}$ chimera. The assays were performed in two independent experiments. Mean (SEM) intracellular (IC, solid line) and extracellular (EC, punctuated line) ATP concentration for S. aureus cells (figure A, grey lines) and S. marcescens cells (figure B, grey lines) treated with chimera 1 compared to MilliQtreated control (black lines). 
We determined ATP leakage from $S$. aureus when treated with chimeras $4 \mathrm{a}, 4 \mathrm{~b}$ and $4 \mathrm{c}$ to evaluate the effect of chain length on the extent of pore formation or membrane disintegration caused by the chimeras. Peptidomimeticinduced ATP leakage was markedly different for $S$. aureus treated with chimera 4a (Figure 4A) as compared to $S$. aureus treated with chimera 4c (Figure 4C). The immediate ATP release was approximately $15 \mu \mathrm{M}$ for both peptidomimetics; however, the intracellular ATP concentration remained at approx. $5 \mu \mathrm{M}$, when the bacterial cells were treated with the shorter analogue $4 \mathrm{a}$, whereas cells treated with chimera 4c were immediately depleted of intracellular ATP. Since the leakage was continuous it seemed that the cells were able to maintain the ATP production. S. aureus cells treated with the intermediate length 12-meric chimera $4 \mathrm{~b}$ had the same leakage pattern as induced by chimera $4 \mathrm{a}$. Dose-response profiles were also determined (as already described in the previous section), and despite differences in MIC values between chimeras $4 \mathrm{a}$ and $4 \mathrm{c}$, both reached the immediate maximum ATP release at $500 \mu \mathrm{g} / \mathrm{mL}$ (i.e. $276 \mu \mathrm{M}$ and $140 \mu \mathrm{M}$, respectively). Likewise, the observed ATP release was similar immediately upon treatment with either chimera $4 \mathrm{a}$ or 4c, and again cells treated with chimera 4a were able to maintain a low intracellular level of ATP.

The fact that some ATP remained in the cell after treatment with chimera 4 a could point to an incomplete disruption of the bacterial cell membrane as compared to bacterial cells treated with chimera 4c. To determine if an intracellular ATP concentration of $5 \mu \mathrm{M}$ had a physiological effect and would allow the bacterial cells to survive, time-kill was again performed under exactly the same conditions as used in the ATP assay to allow comparison of ATP leakage with killing kinetics. After treatment with chimera $4 \mathrm{c}$, cell numbers were reduced with 2 log within the first 20 minutes (Figure 4D), however,

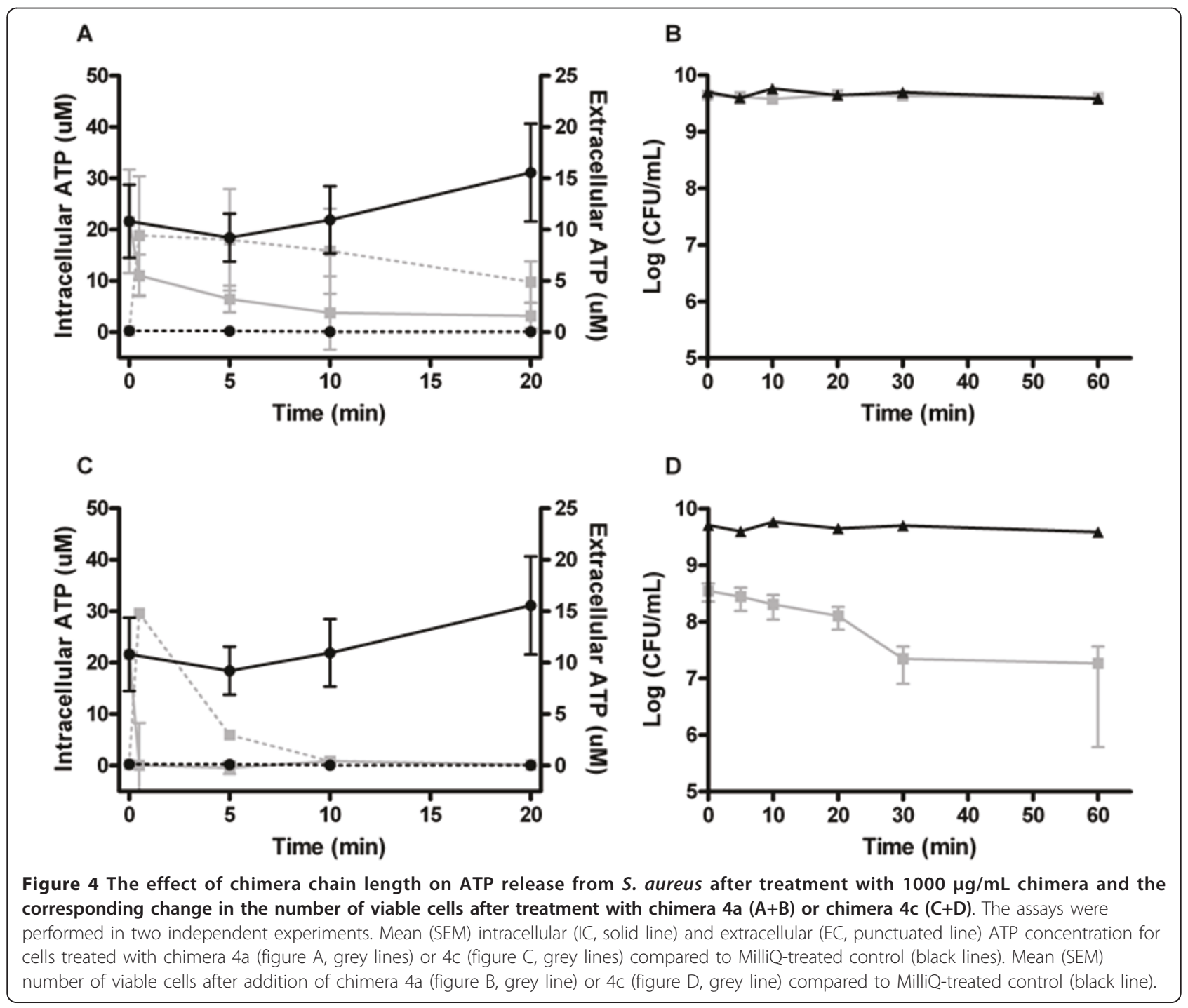


after treatment with chimera 4a (Figure 4B) or chimera 4b (not shown) no killing was observed. The pool of intracellular ATP in the peptidomimetic-treated bacterial cells can therefore, as opposed to the amount of leaked ATP, be considered as indicative for the number of viable cells remaining.

\section{Discussion}

The aim of this study was to determine the mechanism of action for a series of peptidomimetics, and specifically we set out to probe the importance of amino acid composition and chain length for antibacterial activity. We included a strain intrinsically resistant to AMPs, and addressed whether killing kinetics and AMP mechanism of action in viable bacteria could provide a mechanistic explanation for the much lower susceptibility of $S$. marcescens as compared to the more sensitive bacteria.

We examined the effect of having exclusively lysine or homoarginine cationic residues as well as of substituting the chiral $\beta$-peptoids with achiral counterparts as represented by the $\alpha$-peptide/ $\beta$-peptoid chimeras 1,2 and 3 (Table 2). All three peptidomimetics had MIC values of 1$3 \mu \mathrm{M}$ against most bacterial strains, which compared to many natural AMPs is a high activity [14,19,37-39]. Noticeably, a considerably lower activity against $S$. aureus and $K$. pneumoniae was observed for the lysine-containing chimera 3 (6-13 fold) as compared to the homoargininebased chimera 2 , while only a slightly lower activity of chimera 3 (2-7 fold) was seen compared to chimera 2 when tested against E.coli. The reduced chirality in chimera 1 did not give rise to any significant loss of activity as compared to chimera 2 . In a preliminary antimicrobial characterization these peptidomimetics were tested against four common bacteria and a fungus [23], whereas the present study also included important food-borne pathogens $L$. monocytogenes, $V$. vulnificus and $V$. parahaemolyticus against which the chimeras also were active (Table 2).

Additionally we investigated the effect of chain length on activity by studying a series of three peptidomimetics (i.e. chimera $4 \mathrm{a}, 4 \mathrm{~b}$ and $4 \mathrm{c}$ based on the same repeating unit of four residues), which indicated that the minimally required length for an active peptidomimetic is around 12 residues (Table 2). It has previously been reported that 14 amino acids is the minimal sequence required for an active antimicrobial peptide [25], however, this and other studies focused on the effect of length on helicity which implies structural restrictions in the design to enable it to span the lipid bilayer $[26,40]$. Also, it is clearly established that the low activity earlier reported for the shorter homologues of chimera 3 (e.g. the 12-mer exhibited almost no activity [23]) may be compensated for by a longer sequence. Chimera $4 \mathrm{c}$ corresponds to the analogue where half of the lysines in chimera 3 are replaced by homoarginines, and similarly chimera $4 \mathrm{~b}$ may be considered an analogue derived from chimera 2 by exchanging half of the homoarginines with lysines. Comparison of the activities found for these two pairs indicates that a high content of homoarginines generally induces a somewhat higher potency; especially, the activity against $S$. aureus and $K$. pneumoniae is clearly promoted by a prevalence of guaninido-functionalized residues.

A high activity was also found against two isolates of ESBL-producing E. coli (AAS-EC-09 and AAS-EC-010) indicating that resistance towards conventional antibiotics do not affect the sensitivity towards these peptidomimetics, further supporting a different mode of action. Many AMPs exhibit a cell envelope-perturbing effect [41-43], and hence their target is different from traditional antibiotics of which many act by inhibiting cell wall synthesis or on intracellular targets [44-46]. Notably, S. marcescens was the only bacterial strain that proved tolerant to the peptidomimetics, and thus must harbour specific resistance mechanisms involving induction of changes in the cell envelope.

Time-kill experiments showed that $S$. marcescens was killed more rapidly than the susceptible strain of $S$. aureus when treated with chimera 1,2 or 3 at concentrations close to their MIC values (Figure 2). Polymyxin B and other cationic AMPs may at high doses in themselves act like chelating agents allowing them to penetrate the outer membrane $[47,48]$, however, a noticeable effect was also seen against $S$. marcescens at concentrations lower that the MIC value (Figure 2C). Rapid killing was also demonstrated for $E$. coli exposed to the peptidomimetics, indicating that this could be a phenomenon associated with Gram-negative bacteria. Shorter exposure times caused a significant killing of Gram-negative bacteria when treated with some $\alpha$-helical AMPs that act by permeabilization of the membrane [37]. Another explanation for the observed differences in the rate of killing could be that either the degree or mode of membrane disruption differs among bacteria i.e. the chimeras may exert their effect by a combination of several mechanisms. The fact that cell membranes of different bacteria differs in lipid composition [49] could influence the interaction between phospholipids and AMPs. However, there is no unequivocal evidence demonstrating that an AMP may exhibit different pore-forming properties in different bacteria, as the proposed co-existence of several disruption modes in fact still is a topic of debate [50,51].

Many AMPs exert their antibacterial effect by interactions with the bacterial cell membrane $[38,41,52]$ involving pore formation or membrane disintegration that in turn causes leakage of the cell contents, which ultimately leads to cell death. Nevertheless, there is a growing amount of indirect evidence that the mechanisms of some very potent AMPs in fact involves an initial period 
of intracellular accumulation prior to the actual bacterial killing indicating that they act on intracellular targets $[38,53,54]$. To further investigate the effect of the present peptidomimetics on the cell membrane in $S$. marcescens and $S$. aureus and to determine how structural features of these peptidomimetics might affect the potential membrane-related mode of action we examined their ability to cause leakage of intracellular compounds e.g. ATP. A considerable body of data on the leakage of intracellular compounds has already been obtained by using model membranes thus confirming that many membrane-active peptides indeed exert a permeabilizing effect [24-26,28]. These studies have, however, not demonstrated whether there is a direct kinetic relationship between cell membrane damage and loss of viability, and for this reason we combined leakage assays with a time-kill experiment under exactly the same conditions.

Treatment of both $S$. marcescens and $S$. aureus with peptidomimetics 1, 2 and 3 caused leakage of ATP from the bacterial cells with a similar simultaneous reduction in the number of viable cells, and therefore we conclude that even though $S$. marcescens is tolerant to the peptidomimetics their mode of action against this bacterium is similar to that of S. aureus. Earlier, chimera 3 was investigated for its ability to induce calcein leakage in unilamellar liposomes mimicking human cell membranes with a positive response [24], but based on the consistent results in the present work all three peptidomimetics are likely to permeabilize both model and bacterial membranes. Leakage of intracellular compounds has been determined to be the mode of action for many AMPs [55-57], but here we have established this mode of action for a series of peptidomimetics. We conclude that variation of the type of cationic amino acid (i.e. lysine versus homoarginine) did not have an effect on the mode of action in viable bacteria.

Since S. marcescens was tolerant to all peptidomimetics tested, their mode of action must therefore involve a target that is ultimately changed by resistance mechanisms in this species. It is well-known that $S$. marcescens is tolerant to the polymyxin group of antimicrobials, and the main hypothesis is that this is due to inherent changes in the composition of the LPS of the Gram-negative outer membrane that acts as a barrier [33]. We demonstrated that the outer membrane also seems to play an important role in the tolerance of $S$. marcescens towards our chimeras as a combined treatment including the chelating agent EDTA resulted in a reduction in the number of viable cells comparable to that seen for a more susceptible Gram-negative strain of $E$. coli treated similarly (not shown). This indicated that the innate differences in susceptibility between the two Gram-negative species could be completely eliminated after destabilization of the outer membrane.
When designing new antimicrobial peptides it is generally accepted that a minimum length is required in order for the peptide to span or transverse the cell membrane. However, the majority of studies have focused on optimizing the length of AMPs assuming it to adopt a helical conformation $[25,26,40]$. By contrast, due to their design with alternating hydrophobic and cationic residues our peptidomimetics are not expected to adopt an amphipathic helical active confirmation, but rather an extended conformation with some degree of secondary structure as indicated by analysis of their CD spectra [22,23]. Recently, it has been shown that neither global amphipathicity nor regular secondary structure may be required for short peptides to effectively interact with bacterial membranes $[19,58]$, but the optimal length of such peptides has not been rationalized by mechanistic experiments. Only oligomers with a chain length above 12 residues, i.e. the 16 -meric peptidomimetic 4c were able to cause such a substantial leakage of ATP that the number of viable cells were reduced (Figure 4C and 4D). We attribute this to the inability of chimeras $4 \mathrm{a}$ and $4 \mathrm{~b}$ to produce a critical degree of membrane disruption thus leaving a sufficient level of intracellular ATP for the cells to survive (Figure 4A and $4 \mathrm{~B}$ for chimera $4 \mathrm{a}$ ).

This is to our knowledge the first time that the effect of chain length has been investigated on the membraneperturbing activity of peptidomimetics without a dominant secondary structure. Also, we believe that our study is the first that directly, in a kinetic fashion, correlate membrane permeabilization with actual killing kinetics.

Previously, the interaction of $\alpha$-peptide/ $\beta$-peptides chimeras with liposomal model membranes and murine fibroblast was described [24]. Most recently, we investigated their cytotoxicity and haemolytic activity towards human HeLa cells and erythrocytes, respectively [23]. Besides confirming that members of this subclass of peptidomimetics exhibit a broad antimicrobial activity that includes resistant strains and food-borne pathogens, the purpose of the present study was to undertake a more detailed investigation of their mode of action. The present contribution describes their interaction with viable bacterial cells, and we found that these antimicrobial peptidomimetics have a mode of action involving the cell membrane. The observed membrane disruption depends strongly on chain length, and it may be impeded if the outer membrane in a Gram-negative bacterium possesses an innate altered composition.

\section{Conclusion}

Several $\alpha$-peptide/ $\beta$-peptoid chimeras were bactericidal against important food-borne and clinical pathogens with MIC values in the range of 1-5 $\mu \mathrm{M}$. We examined 
the effect of changing the ratio between amino- and guanidino-functionalized cationic residues as well as the influence of chain length on both antibacterial activity and ATP leakage. Although, minor differences in the antimicrobial profile of the chimeras may be ascribed to the degree of chirality and/or type of cationic amino acids, by far the most pronounced impact stems from the chain length. Only one bacterial species, S. marcescens, was tolerant to the peptidomimetics most likely due to the composition of its outer membrane; however, the ATP leakage was as pronounced as seen for more sensitive bacteria. We conclude that these synthetic antimicrobial peptidomimetics exert their effect through permeabilization of the cell membrane, and that this corresponds to a simultaneous reduction in the number of viable bacteria with the pool of intracellular ATP being indicative of viability. This is the first time that a relationship is established between permeabilization and killing within a peptidomimetics library.

\section{Acknowledgements}

LHK was funded by a Ph.D. grant from the Technical University of Denmark and the Danish Research Council for Technology and Production (grant number 09-065902/FTP). The authors wish to thank the National Center for Antimicrobials \& Infection Control, Statens Serum Institut, Denmark for providing the Danish clinical samples of ESBL-producing E. coli. We thank, the Brødrene Hartmanns Fond (Copenhagen) for a materials grant supporting the synthesis work.

\section{Author details}

'Division of Industrial Food Research, National Food Institute, Technical University of Denmark, Søltofts Plads, bldg.221, 2800 Kgs. Lyngby,

DK-Denmark. ${ }^{2}$ Department of Medicinal Chemistry, Faculty of Pharmaceutical Science, University of Copenhagen, Universitetsparken 2, 2100 København $\varnothing$, DK-Denmark.

\section{Authors' contributions}

LHK planned and carried out all experiments and drafted the manuscript. HF designed the peptidomimetics and participated in the revision of the manuscript. KMK synthesized the peptidomimetics. LG helped in the design of the experiments and the drafting of the manuscript. All authors have seen and approved the final manuscript.

\section{Competing interests}

The authors declare that they have no competing interests.

Received: 30 March 2011 Accepted: 22 June 2011

Published: 22 June 2011

\section{References}

1. Zasloff M: Antimicrobial peptides of multicellular organisms. Nature 2002, 415:389-395.

2. Bowdish DM, Davidson DJ, Lau YE, Lee K, Scott MG, Hancock RE: Impact of LL-37 on anti-infective immunity. J Leukoc Biol 2005, 77:451-459.

3. Ganz T: Defensins: antimicrobial peptides of innate immunity. Nat Rev Immunol 2003, 3:710-720

4. Gallo RL, Nizet V: Endogenous production of antimicrobial peptides in innate immunity and human disease. Curr Allergy Asthma Rep 2003, 3:402-409.

5. Brown KL, Hancock RE: Cationic host defense (antimicrobial) peptides. Curr Opin Immunol 2006, 18:24-30.

6. Boucher HW, Talbot GH, Bradley JS, Edwards JE, Gilbert D, Rice LB, et al: Bad bugs, no drugs: no ESKAPE! An update from the Infectious Diseases Society of America. Clin Infect Dis 2009, 48:1-12.
7. Fischbach MA, Walsh CT: Antibiotics for emerging pathogens. Science 2009, 325:1089-1093.

8. Hancock RE, Sahl HG: Antimicrobial and host-defense peptides as new anti-infective therapeutic strategies. Nat Biotechnol 2006, 24:1551-1557.

9. Chen Y, Mant CT, Farmer SW, Hancock RE, Vasil ML, Hodges RS: Rational design of a-helical antimicrobial peptides with enhanced activities and specificity/therapeutic index. J Biol Chem 2005, 280:12316-12329.

10. Chongsiriwatana NP, Patch JA, Czyzewski AM, Dohm MT, Ivankin A, Gidalevitz D, et al: Peptoids that mimic the structure, function, and mechanism of helical antimicrobial peptides. Proc Natl Acad Sci USA 2008, 105:2794-2799.

11. Oren Z, Shai Y: Selective lysis of bacteria but not mammalian cells by diastereomers of melittin: structure-function study. Biochemistry 1997, 36:1826-1835.

12. Patch JA, Barron AE: Mimicry of bioactive peptides via non-natural, sequence-specific peptidomimetic oligomers. Curr Opin Chem Biol 2002, 6:872-877.

13. Porter EA, Weisblum B, Gellman SH: Mimicry of host-defense peptides by unnatural oligomers: antimicrobial $\beta$-peptides. J Am Chem Soc 2002, 124:7324-7330.

14. Radzishevsky IS, Kovachi T, Porat Y, Ziserman L, Zaknoon F, Danino D, et al: Structure-activity relationships of antibacterial acyl-lysine oligomers. Chem Biol 2008, 15:354-362.

15. Raventos D, Taboureau O, Mygind PH, Nielsen JD, Sonksen CP, Kristensen HH: Improving on nature's defenses: optimization \& high throughput screening of antimicrobial peptides. Comb Chem High Throughput Screen 2005, 8:219-233.

16. Perez-Paya E, Houghten RA, Blondelle SE: The role of amphipathicity in the folding, self-association and biological activity of multiple subunit small proteins. J Biol Chem 1995, 270:1048-1056.

17. Powers JP, Hancock RE: The relationship between peptide structure and antibacterial activity. Peptides 2003, 24:1681-1691.

18. Schmitt MA, Weisblum B, Gellman SH: Unexpected relationships between structure and function in $\alpha, \beta$-peptides: antimicrobial foldamers with heterogeneous backbones. J Am Chem Soc 2004, 126:6848-6849.

19. Schmitt MA, Weisblum B, Gellman SH: Interplay among folding, sequence, and lipophilicity in the antibacterial and hemolytic activities of $\alpha / \beta$ peptides. J Am Chem Soc 2007, 129:417-428.

20. Tossi A, Tarantino C, Romeo D: Design of synthetic antimicrobial peptides based on sequence analogy and amphipathicity. Eur J Biochem 1997, 250:549-558.

21. Bonke G, Vedel L, Witt M, Jaroszewski JW, Olsen CA, Franzyk H: Dimeric building blocks for solid-phase synthesis of a-peptide- $\beta$-peptoid chimeras. Synthesis 2008, 15:2381-2390.

22. Olsen CA, Bonke G, Vedel L, Adsersen A, Witt M, Franzyk $H$, et al: $\boldsymbol{\alpha}$ peptide/ß-peptoid chimeras. Org Lett 2007, 9:1549-1552.

23. Olsen CA, Ziegler HL, Nielsen HM, Frimodt-Moller N, Jaroszewski JW, Franzyk H: Antimicrobial, hemolytic, and cytotoxic activities of $\beta$-peptoidpeptide hybrid oligomers: improved properties compared to natural AMPs. Chembiochem 2010, 11:1356-1360.

24. Foged C, Franzyk H, Bahrami S, Frokjaer S, Jaroszewski JW, Nielsen HM, et al: Cellular uptake and membrane-destabilising properties of a-peptide/ $\beta$ peptoid chimeras: lessons for the design of new cell-penetrating peptides. Biochim Biophys Acta 2008, 1778:2487-2495.

25. Javadpour MM, Juban MM, Lo WC, Bishop SM, Alberty JB, Cowell SM, et al: De novo antimicrobial peptides with low mammalian cell toxicity. J Med Chem 1996, 39:3107-3113.

26. Agawa $Y$, Lee $S$, Ono $S$, Aoyagi $H$, Ohno $M$, Taniguchi $T$, et al: Interaction with phospholipid bilayers, ion channel formation, and antimicrobial activity of basic amphipathic a-helical model peptides of various chain lengths. J Biol Chem 1991, 266:20218-20222.

27. Zhang L, Rozek A, Hancock RE: Interaction of cationic antimicrobial peptides with model membranes. J Biol Chem 2001, 276:35714-35722.

28. Yu L, Guo L, Ding JL, Ho B, Feng SS, Popplewell J, et al: Interaction of an artificial antimicrobial peptide with lipid membranes. Biochim Biophys Acta 2009, 1788:333-344.

29. Vedel L, Bonke G, Foged C, Ziegler H, Franzyk H, Jaroszewski JW, et al: Antiplasmodial and prehemolytic activities of a-peptide- $\beta$-peptoid chimeras. Chembiochem 2007, 8:1781-1784. 
30. The Clinical and Laboratory Standards Institute (CLSI): Guideline M7-A7: Methods for dilution antimicrobial susceptibility tests for bacteria that grow aerobically 2006, Approved Standard Seventh Edition.

31. Johansen C, Verheul A, Gram L, Gill T, Abee T: Protamine-induced permeabilization of cell envelopes of gram-positive and gram-negative bacteria. Appl Environ Microbiol 1997, 63:1155-1159.

32. Kubitschek HE, Friske JA: Determination of bacterial cell volume with the Coulter Counter. J Bacteriol 1986, 168:1466-1467.

33. Brown DA, Tsang JC: Chemical and electrophoretic changes induced by polymyxin B on outer membrane components from Serratia marcescens. J Antibiot (Tokyo) 1978, 31:603-609.

34. McCoy AJ, Liu H, Falla TJ, Gunn JS: Identification of Proteus mirabilis mutants with increased sensitivity to antimicrobial peptides. Antimicrob Agents Chemother 2001, 45:2030-2037.

35. Anisimov AP, Dentovskaya SV, Titareva GM, Bakhteeva IV, Shaikhutdinova RZ, Balakhonov SV, et al: Intraspecies and temperaturedependent variations in susceptibility of Yersinia pestis to the bactericidal action of serum and to polymyxin B. Infect Immun 2005, 73:7324-7331

36. Nummila K, Kilpelainen I, Zahringer U, Vaara M, Helander IM: Lipopolysaccharides of polymyxin B-resistant mutants of Escherichia coli are extensively substituted by 2-aminoethyl pyrophosphate and contain aminoarabinose in lipid A. Mol Microbiol 1995, 16:271-278.

37. Giangaspero A, Sandri L, Tossi A: Amphipathic a helical antimicrobial peptides. Eur J Biochem 2001, 268:5589-5600.

38. Rotem S, Radzishevsky IS, Bourdetsky D, Navon-Venezia S, Carmeli Y, Mor A: Analogous oligo-acyl-lysines with distinct antibacterial mechanisms. FASEB J 2008, 22:2652-2661

39. Chou HT, Kuo TY, Chiang JC, Pei MJ, Yang WT, Yu HC, et al: Design and synthesis of cationic antimicrobial peptides with improved activity and selectivity against Vibrio spp. Int J Antimicrob Agents 2008, 32:130-138.

40. Deslouches B, Phadke SM, Lazarevic V, Cascio M, Islam K, Montelaro RC, et al: De novo generation of cationic antimicrobial peptides: influence of length and tryptophan substitution on antimicrobial activity. Antimicrob Agents Chemother 2005, 49:316-322.

41. Wu M, Hancock RE: Interaction of the cyclic antimicrobial cationic peptide bactenecin with the outer and cytoplasmic membrane. J Biol Chem 1999, 274:29-35.

42. Phadke SM, Lazarevic V, Bahr CC, Islam K, Stolz DB, Watkins S, et al: Lentivirus lytic peptide 1 perturbs both outer and inner membranes of Serratia marcescens. Antimicrob Agents Chemother 2002, 46:2041-2045.

43. Loit E, Hincke MT, Altosaar I: Synthetic antimicrobial peptide L8 (MHLHKTSRVTLYLL) has membrane permeabilisation and bacterial aggregation activity. Int J Antimicrob Agents 2010, 35:410-411.

44. Harms JM, Bartels $H$, Schlunzen F, Yonath A: Antibiotics acting on the translational machinery. J Cell Sci 2003, 116:1391-1393.

45. Schmitz FJ, Higgins PG, Mayer S, Fluit AC, Dalhoff A: Activity of quinolones against gram-positive cocci: mechanisms of drug action and bacterial resistance. Eur J Clin Microbiol Infect Dis 2002, 21:647-659.

46. Reynolds PE: Structure, biochemistry and mechanism of action of glycopeptide antibiotics. Eur J Clin Microbiol Infect Dis 1989, 8:943-950.

47. Tsang JC, Weber DA, Brown DA: Evidences for complex formation between polymyxin B and lipopolysaccharides from Serratia marcescens. J Antibiot (Tokyo) 1976, 29:735-742.

48. Hancock RE: The bacterial outer membrane as a drug barrier. Trends Microbiol 1997, 5:37-42.

49. Epand RM, Epand RF: Bacterial membrane lipids in the action of antimicrobial agents. J Pept Sci 2010.

50. Hancock RE, Chapple DS: Peptide antibiotics. Antimicrob Agents Chemother 1999, 43:1317-1323.

51. Bechinger B: The structure, dynamics and orientation of antimicrobial peptides in membranes by multidimensional solid-state NMR spectroscopy. Biochim Biophys Acta 1999, 1462:157-183.

52. Koo SP, Yeaman MR, Nast CC, Bayer AS: The cytoplasmic membrane is a primary target for the staphylocidal action of thrombin-induced platelet microbicidal protein. Infect Immun 1997, 65:4795-4800.

53. Schneider T, Kruse T, Wimmer R, Wiedemann I, Sass V, Pag U, et al: Plectasin, a fungal defensin, targets the bacterial cell wall precursor Lipid II. Science 2010, 328:1168-1172.
54. Casteels $P$, Tempst $P$ : Apidaecin-type peptide antibiotics function through a non-poreforming mechanism involving stereospecificity. Biochem Biophys Res Commun 1994, 199:339-345.

55. Zaknoon F, Sarig H, Rotem S, Livne L, Ivankin A, Gidalevitz D, et al: Antibacterial properties and mode of action of a short acyl-lysyl oligomer. Antimicrob Agents Chemother 2009, 53:3422-3429.

56. Guihard G, Benedetti H, Besnard M, Letellier L: Phosphate efflux through the channels formed by colicins and phage T5 in Escherichia coli cells is responsible for the fall in cytoplasmic ATP. J Biol Chem 1993, 268:17775-17780.

57. Park SC, Kim JY, Jeong C, Yoo S, Hahm KS, Park Y: A plausible mode of action of pseudin-2, an antimicrobial peptide from Pseudis paradoxa. Biochim Biophys Acta 2011, 1808:171-182.

58. Mondal J, Zhu X, Cui Q, Yethiraj A: Sequence-dependent interaction of $\beta$ peptides with membranes. J Phys Chem B 2010, 114:13585-13592.

59. Novick R: Properties of a cryptic high-frequency transducing phage in Staphylococcus aureus. Virology 1967, 33:155-166.

60. Bachmann BJ: Pedigrees of some mutant strains of Escherichia coli K-12. Bacteriol Rev 1972, 36:525-557.

61. Larsen CN, Norrung B, Sommer HM, Jakobsen M: In vitro and in vivo invasiveness of different pulsed-field gel electrophoresis types of Listeria monocytogenes. Appl Environ Microbiol 2002, 68:5698-5703.

62. Wulff G, Gram L, Ahrens P, Vogel BF: One group of genetically similar Listeria monocytogenes strains frequently dominates and persists in several fish slaughter- and smokehouses. Appl Environ Microbiol 2006, 72:4313-4322.

doi:10.1186/1471-2180-11-144

Cite this article as: Hein-Kristensen et al:: Bacterial membrane activity of $\alpha$-peptide/ $\beta$-peptoid chimeras: Influence of amino acid composition and chain length on the activity against different bacterial strains. BMC Microbiology 2011 11:144.

\section{Submit your next manuscript to BioMed Central and take full advantage of:}

- Convenient online submission

- Thorough peer review

- No space constraints or color figure charges

- Immediate publication on acceptance

- Inclusion in PubMed, CAS, Scopus and Google Scholar

- Research which is freely available for redistribution

Submit your manuscript at www.biomedcentral.com/submit
C) Biomed Central 